\title{
A Case of Refractory Acute Angle Closure Glaucoma after an Intravit- real Bevacizumab Injection
}

\section{Dear Editor,}

Intravitreal injection is a commonly performed and relatively safe procedure for different retinal diseases [1,2]. In most cases, intraocular pressure (IOP) elevation after an intravitreal injection appears as a transient IOP spike due to volume effects in the closed intravitreal cavity. However, IOP elevation due to morphological changes in the anterior chamber $(\mathrm{AC})$ angle has been rarely reported [3,4]. We report a case of acute angle closure (AAC) refractory to mannitol and laser iridotomy that occurred after intravitreal bevacizumab (IVB) injection in an eye for treatment of central retinal vein occlusion.

A 61-year-old man presented with decreased visual acuity (VA) in the right eye that began 1 month prior to admission. The VA of the right eye was $20 / 200$. On slit-lamp examination, the AC was deep and clean, and no definite lens abnormality was observed. Color fundus photography revealed diffuse flame-shaped hemorrhages throughout the whole retina (Fig. 1A). Fluorescein angiography revealed delayed arteriovenous transit time, increased tortuosity of vessels, and a peripheral nonperfusion area (Fig. 1B). On optical coherence tomography of the macula, macular edema was observed (Fig. 1C). The patient was diagnosed with central retinal vein occlusion, and IVB was performed in the right eye.

Macular edema decreased 1 month after injection. Thus, a second IVB was performed. The IOP before the second injection was $18 \mathrm{mmHg}$. Two days after the second injection, the patient reported ocular pain, headache, and nausea, and the VA decreased to hand motion. The IOP in the right eye was $50 \mathrm{mmHg}$. Medication was denied before and after the injection. The $\mathrm{AC}$ was shallow with less than

Received: July 6, 2020 Final revision: July 15, 2020

Accepted: July 31, 2020

$1 / 4$ central corneal thickness at the periphery and approximately 2 central corneal thickness at the center. The pupil was mid-dilated to $7 \mathrm{~mm}$ and fixed. Gonioscopy revealed a closed angle of $360^{\circ}$ without any peripheral anterior synechia or neovascularization (Fig. 1D, 1E). A diagnosis of AAC was made, and $200 \mathrm{~mL}$ of $20 \%$ mannitol was injected intravenously. The IOP did not decrease after administration of intravenous mannitol. Laser iridotomy was performed, but the AC did not deepen nor did the IOP drop. Emergency cataract surgery was performed on the right eye. The axial length of the right eye was $23.29 \mathrm{~mm}$, and
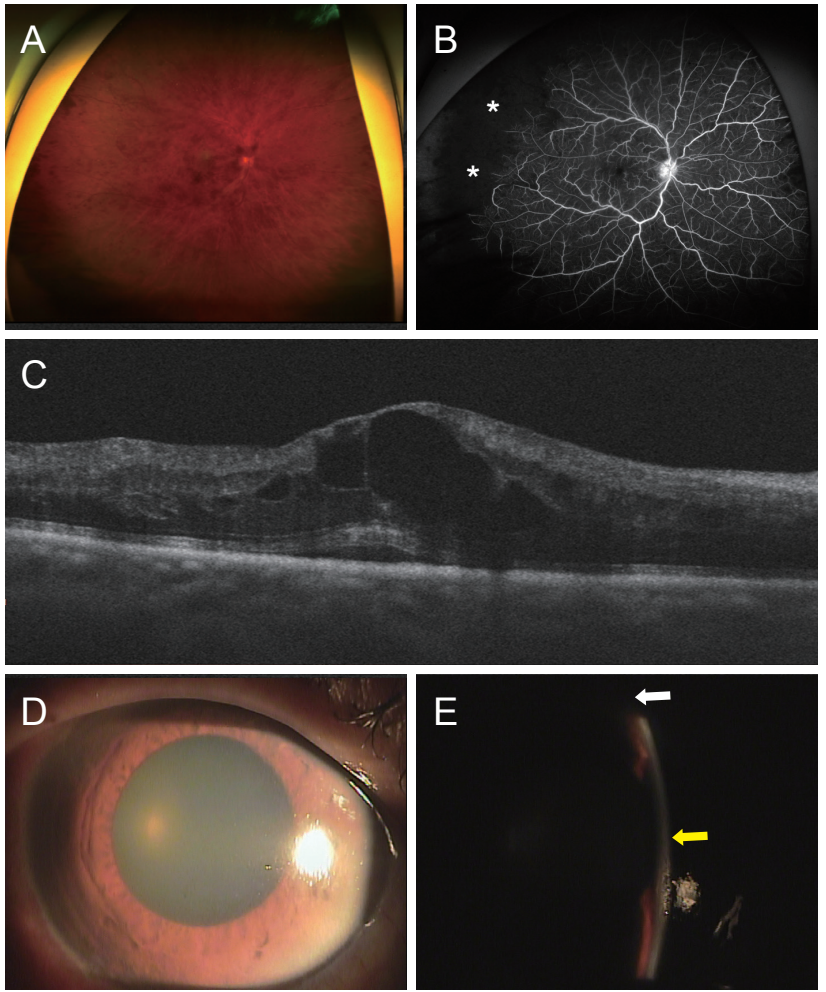

Fig. 1. Multimodal images of the patient. (A) Ultra-widefield (UWF) color fundus photography reveals diffuse flame-shaped hemorrhages throughout whole retina. (B) Ultra-widefield fluorescein angiography shows increased tortuosity of vessels and peripheral nonperfusion area (asterisks). (C) Optical coherence tomography of the macula reveals cystoid macular edema. (D) Slit-lamp examination reveals an edematous cornea with some microbullae. The pupil was mid-dilated to $7 \mathrm{~mm}$ and fixed. (E) The anterior chamber was shallow with less than $1 / 4$ central corneal thickness at the periphery (white arrow) and about 2 central corneal thickness at the center (yellow arrow). The patient provided written informed consent for the publication and the use of images.

\section{(C) 2020 The Korean Ophthalmological Society}

This is an Open Access article distributed under the terms of the Creative Commons Attribution Non-Commercial License (http://creativecommons.org/licenses /by-nc/3.0/) which permits unrestricted non-commercial use, distribution, and reproduction in any medium, provided the original work is properly cited. 
about $100^{\circ}$ zonulysis at temporal with total zonular weakness were observed during the cataract surgery. After phacoemulsification and posterior chamber lens implantation, the IOP decreased to $21 \mathrm{mmHg}$.

Jeong et al. [3] and Semoun et al. [4] previously reported $\mathrm{AAC}$ after IVB. In these cases, the eyes were hyperopic and showed shallow $\mathrm{AC}$ at baseline, accounting for the risk of AAC. The IOP responded to medical treatment, that is, administration of intravenous mannitol or acetazolamide. The mechanism of AAC after intravitreal injection is yet to be elucidated. By using anterior segment optical coherence tomography, Alkin et al. [5] demonstrated that AC depth decreased immediately after intravitreal injection, and normalized to the baseline level with time. This suggests anterior displacement of the iris and lens diaphragm. This may narrow the anterior angle and result in further obstruction of the aqueous humor outflow pathway, especially in a hyperopic patient with a shallow angle, as in previous cases.

However, in our case, the patient was neither hyperopic nor had a shallow AC at initial examination, and the IOP was not controlled by medical treatment. Zonulysis was observed during cataract surgery. Increased posterior volume after IVB may have pressured the lens, and the lens was abnormally displaced anteriorly due to the loosened zonule. Mydriasis before the injection might have exacerbated the situation. Therefore, anterior displacement of the lens blocked the angle, and mannitol or iridotomy could not reverse the situation. Successful phacoemulsification with posterior chamber lens implantation may have reduced the volume of the lens and reduced the burden on the zonule, thereby leading to a deepening of the $\mathrm{AC}$ and a normalization of the IOP in this case.

This was a rare case of AAC after IVB in an eye with zonulysis resolved with cataract surgery. Although AAC is a rare complication after intravitreal injection, it can occur not only in eyes with shallow ACs but also in eyes with zonular weaknesses. Therefore, this should be considered in AAC cases after intravitreal injection, and thorough anterior segment examination before an injection is recommended.

In Kee Kim, Jiwon Baek

Department of Ophthalmology, Bucheon St. Mary's Hospital, The Catholic University of Korea College of Medicine, Bucheon, Korea

E-mail (Jiwon Baek):20900102@cmcnu.or.kr

\section{Conflict of Interest}

No potential conflict of interest relevant to this article was reported.

\section{References}

1. Lai TY, Liu S, Das S, Lam DS. Intravitreal injection--technique and safety. Asia Pac J Ophthalmol (Phila) 2015;4:3218.

2. Jain P, Sheth J, Anantharaman G, Gopalakrishnan M. Real-world evidence of safety profile of intravitreal bevacizumab (Avastin) in an Indian scenario. Indian J Ophthalmol 2017;65:596-602.

3. Jeong S, Sagong M, Chang W. Acute angle closure attack after an intravitreal bevacizumab injection for branch retinal vein occlusion: a case report. BMC Ophthalmol 2017;17:25.

4. Semoun O, Blumen-Ohana E, de Preobrajensky N, Nordmann JP. Acute angle-closure glaucoma complicating an intravitreal injection of bevacizumab. J Fr Ophtalmol 2009;32:58.

5. Alkin Z, Perente I, Altan C, et al. Changes in anterior segment morphology after intravitreal injection of bevacizum$\mathrm{ab}$ and bevacizumab-triamcinolone acetate combination. Eur J Ophthalmol 2013;23:504-9. 